\title{
BROAD-BAND DOA ESTIMATION AND BEAMFORMING IN MULTIPATH ENVIRONMENT
}

\author{
K. C. Indukumar and V. U. Reddy
}

Department of Electrical Communication Engineering

Indian Institute of Science, Bangalore - 560 012, INDIA

\begin{abstract}
Recently, Buckley [ 1) has developed orthogonal low-dimension models for broad-band sources and used them in $\boldsymbol{D O A}$ ( direction of arrival ) estimation and beamforming. The dimensionality of this lowdimension model is the number of significant eigenvectors of the source sample covariance matrix. When two or more sources impinge on the array, the dimensionality of the composite signal subspace is taken to be the sum of the dimensions of individual source representation spaces. This is the case when the sources are uncorrelated.

In this paper, considering a two-source model, we first show that the dimensionality of the composite signal subspace collapses when the signals from these sources are correlated, as it happens in the case of multipath, and the extent by which it collapses depends on the degree of correlation. Using the F-norm (Robenius norm ) of the crosscorrelation matrix of the direct and multipath signals as a measure of correlation between them, we illustrate how the norm decrease as the delay increases, indicating that the degree of correlation falls with delay. We propose spatial smoothing for reducing the norm and show that the norm decreases to zero asymptotically with the number of subarrays. We then derive an analytical expression for an upper bound on the F-norm and using this, we study the effect of spacing an directions of the sources on the rate of decorrelation with progressive smoothing

Simulations are provided to illustrate the impact of progressive smoothing on $\boldsymbol{D O A}$ estimation and beamforming performance.
\end{abstract}

\section{INTRODUCTION}

Subspace based methods of $\boldsymbol{D O A}$ ( direction of arrival ) estimation, pioneered by Pisarenko [ $\mathbf{2}$ ], and generalised by Schmid [ 3 ] and Bienvenu [ 4 ] for narrowband signals, have been recently extended to broad-band case by Buckley [ 1,5], Buckley and Griffiths [ 6 ) and, Wang and Kaveh [ 7]. In [1], Buckley has developed lowdimension models for the broad-band sources, termed as broad-band representation spaces, and used these models in $\boldsymbol{D O A}$ estimation and beamforming. As defined in $[1]$, the dimensionality of the broad-band source representation space is the number of significant eigenvectors of the source sample covariance matrix, which may also be viewed as the effective rank of this matrix. When several sources impinge on the array, the dimensionality of the composite signal subspace, which represents all the impinging sources, is taken to be the sum of the dimensions of the individual source representation spaces. This implicitly assumes that the impinging signals are uncorrelated.

There are situations when several or all of the impinging signals are correlated, e.g., multipath propagation and smart jamming environments. In multipath propagation, the signal arrives via a direct and reflected paths and the DOAs of these signals are in general different. The signal arriving via the reflected path (which we refer to as the multipath signal) can be viewed as the signal emitted from another source that is correlated with the one associated with the direct path. The delay between the two signals, i.e., the difference between the times of arrival of the direct and reflected signals, as ob-

This work is supported in part by Electronics and Radar Development Establishment, Bangalore 560 093, INDIA

$\mathrm{CH} 2882-9 / 90 / 0000-0532 \$ 1.00$ (C) (1990 IEEE) served at the reference sensor of the array, depends on the path lengths of these two signals. Recently, the $\boldsymbol{D O \boldsymbol { A }}$ estimation performance of the subspace-based methods and the performance of the linearly constrained $M V$ ( minimum variance) beamformer in the presence of multipath have been extensively analysed in the case of narrowband signals $[8,9,10,11]$. In particular, subarray averaging has been proposed in [ 8] and [9], and the impact of progressive smoothing (averaging with increasing number of subarrays ) has been analysed in $[\mathbf{1 0}]$ and $[\mathbf{1 1}]$.

In this paper, we first show that in the presence of multipath, the dimensionality of the composite signal subspace collapses and the extent by which it collapses depends on the amount of multipath delay. We show this by choosing a two-source model with the two sources corresponding to the signals arriving via the direct and reflected paths. We use the F-norm (Robenius norm ) of the cross-correlation matrix of the data sample vectors of these signals as a measure of correlation between them and show that this norm decreases as the delay between the two signals increases. Next, we show that the dimensionality of the signal subspace is restored with subarray averaging. However, the restoration of this dimensionality does not mean that the correlation between the two signals has reduced to zero. The significant residual correlation which is still left between these signals affects the $D O A$ estimation performance in finite data case and interference rejection performance of the $M V$ beamformer even in the infinite data case. We illustrate this with a few examples in Section 5

To study the effect of progressive smoothing on the correlation, we derive an expression for the upper bound on the F-norm of the crosscorrelation matrix which shows that the norm tends to zero asymptotically with the number of subarrays indicating that the correlation falls to zero only asymptotically. The effect of spacing and direction of the sources on the rate of decorrelation with progressive smoothing is also broughtout by this bound. Simulation results are provided to support our assertions.

We may point out here that we chose the two-source signal model to keep the notation and the analysis simple. The results, however, extend to arbitrary number of sources.

\section{BACKGROUND}

Consider a linear ${ }^{\mathbf{1}}$ array with Misotropic sensors and a tapped delay line with K taps attatched to each sensor (see Fig. 1). Assume that two broad-band sources are impinging on the array from two directions $\theta_{d}$ and $\theta_{s}$, and let $d(t)$ and $s(t)$ be the signals emitted by these sources. Let $\mathrm{X}(t)$ denote the $K M$ dimensional stacked vector formed from $\mathrm{K}$ delayed array output vectors as given below

$$
\mathbf{X}(t)=\left[\chi_{1}^{T}(t), \chi_{2}^{T}(t), \ldots, \chi_{K}^{T}(t)\right]^{T}
$$

where

$$
\chi_{k}(t)=\left[x_{1 k}(t), x_{2 k}(t), \ldots, x_{M k}(t)\right]^{T}
$$

'Though the assumption of linear array is not required in obtaining some results of the paper such as, collapse of signal subspace dimensionality in the presence of multipath and the interpretation of the F-norm of cross-correlation matrix as a measure of the correlation, it is essential for spatial smoothing and therefore, we assume a linear array through out the paper. 
Here, $\boldsymbol{x}_{\boldsymbol{m} \boldsymbol{k}}(t)$ denotes the signal from element $\mathrm{m}$ at k-th tap at time instant $t$. The vector $\mathbf{X}(\mathrm{t})$ is also termed as the array snapshot vector at time $t$. We can decompose $x_{m k}(t)$ as

$$
x_{m k}(t)=d_{m k}(t)+s_{m k}(t)+v_{m k}(t)
$$

where $\boldsymbol{d}_{m k}(t)$ and $\boldsymbol{s}_{m k}(t)$ are the components due to the signals arriving from $\theta_{d}$ and $\theta_{s}$, respectievely, and $v_{m k}(t)$ is the component due to the additive sensor noise which is assumed to be uncorrelated with the sources and also to be spatially white. Assuming the first sensor element to be the reference element, we can express

$$
\begin{aligned}
& d_{m k}(t)=d\left(t-(m-1) T_{d}-(k-1) T_{o}\right) \\
& s_{m k}(t)=s\left(t-(m-1) T_{s}-(k-1) T_{o}\right)
\end{aligned}
$$

where $T_{d}$ and $T_{\boldsymbol{d}}$ are the inter-element propagation delays

$$
T_{d}=\frac{A}{\mathrm{c}} \sin \theta_{d}, \quad T_{s}=\frac{\mathrm{A}}{c} \sin \theta_{s}
$$

with $\boldsymbol{A}$ denoting the element spacing and c denoting the velocity of propagation, and $T_{o}$ is the delay between taps. Since the noise is assumed to be independent from sensor to sensor, we can express

$$
v_{m k}(t)=v_{m}\left(t-(k-1) T_{o}\right)
$$

The MK by MK sample data covariance matrix is given by

$$
\Phi=E\left[\mathbf{X}(t) \mathbf{X}^{+}(t)\right]
$$

which, in view of (2.3), can be expressed as

$$
\Phi=\Phi_{d d}+\Phi_{s s}+\Phi_{d s}+\Phi_{s d}+\Phi_{v v}
$$

where $\boldsymbol{\Phi}_{d d}, \boldsymbol{\Phi}_{\boldsymbol{s} s}$ and $\boldsymbol{\Phi}_{\boldsymbol{v} v}$ refer to the sample covariance matrices of source signals and the noise. From (2.1) and (2.2), it follows that $\Phi$ can be partitioned into $K^{2}$ submatrices, each of $M \times \mathrm{M}$ elements. Each submatrix represents the covariance matrix $E\left[\chi_{k}(t) \chi_{j}^{+}(t)\right], 1 \leq$ $k, j \leq K$, where $\chi_{k}(t)$ is a $M \cdot$ dimensional array output vector. We refer to this as the $\boldsymbol{k} \boldsymbol{j}-\boldsymbol{t h}$ submatrix.

In view of (2.2) and (2.3), we can express the mn-th element in kj-th submatrix of $\boldsymbol{\Phi}_{d d}$ as

$$
\left[\Phi_{d d_{m n}}\right]_{k j}=R_{d d}\left((n-m) T_{d}+(j-k) T_{o}\right)
$$

Similarly, we have

$$
\left[\Phi_{s s_{m n}}\right]_{k j}=R_{s s}\left((n-m) T_{s}+(j-k) T_{o}\right)
$$

and

$$
\left[\Phi_{d d_{m n}}\right]_{k j}=R_{d s}\left((n-1) T_{s}-(m-1) T_{d}+(j-k) T_{o}\right)
$$

with

$$
\left[\Phi_{s_{m n}}\right]_{k j}=\left[\Phi_{d s_{n m}}\right]_{j k}^{*}
$$

The last relation follows from the fact that $\boldsymbol{\Phi}_{\boldsymbol{s}}$ is Hermetian transpose of $\boldsymbol{\Phi}_{d s}$, i.e., $\boldsymbol{\Phi}_{s d}=\boldsymbol{\Phi}_{d s}^{+}$. Finally, the mn-th element in kj-th submatrix of $\Phi_{u v}$ is given by

$$
\left[\Phi_{v v_{m n}}\right]_{k j}=\left\{\begin{array}{cc}
R_{v v}\left((j-k) T_{o}\right) & , m=n \\
0 & , m \neq n
\end{array}\right.
$$

If $d(t)$ and $s(t)$ are the signals from uncorrelated sources , the crosscovariance matrices @\& and $\boldsymbol{\Phi}_{a d}$ vanish. In multipath environment, however, one signal is a delayed version of the other, assuming a pure delay environment. If we consider $s(t)$ to be the multipath signal with path delay $T_{\alpha}$, we have

$$
s(t)=d\left(t-T_{\alpha}\right)
$$

The elements of $\boldsymbol{\Phi}_{d s}$ ( and also of $\boldsymbol{\Phi}_{\boldsymbol{s d}}$ ) are then modified as

$$
\left[\Phi_{d o_{m n}}\right]_{k j}=R_{d d}\left(T_{\alpha}+(n-1) T_{s}-(m-1) T_{d}+(j-k) T_{0}\right)
$$

We now assume that $d(t), s(t)$ and $v(t)$ are all zero mean, bandlimited random processes with flat power spectral densities over a band width $\Delta \omega$ centered at frequency $\omega_{0}$. Let the powers in these signals be denoted by $\boldsymbol{\sigma}_{d}^{\mathbf{2}}, \boldsymbol{\sigma}_{\boldsymbol{s}}^{\mathbf{2}}$ and $\boldsymbol{\sigma}_{v}^{2}$, respectievely. Then

$$
R_{d d}(\tau)=\sigma_{d}^{2} \sin c\left(\frac{\Delta \omega \tau}{2}\right) \exp \left(i \omega_{o} \tau\right)
$$

and, $R_{s s}(\tau)$ and $R_{v v}(\tau)$ are same as (2.17) with $\sigma_{d}^{2}$ replaced by $\sigma_{s}^{2}$ and $\sigma_{v}^{2}$, respectievely, and

$$
R_{d e}(\tau)=\sigma_{d} \sigma_{s} \operatorname{sinc}\left(\frac{\Delta \omega \tau}{2}\right) \exp \left(i \omega_{o} \tau\right)
$$

where $\sin c(\cdot)$ denotes the sinc function

$$
\operatorname{sinc}(\alpha)=\frac{\sin \alpha}{\alpha}
$$

and $i$ in the exponent denotes $\sqrt{-1}$. Following Compton [ 12 ], we express the correlation functions in terms of normalised parameters.

Denote the relative bandwidth of the signals as $\boldsymbol{B}_{\boldsymbol{d}}$, i.e., $\boldsymbol{B}_{\boldsymbol{d}}=$ $\Delta \omega / \omega_{o}$, and express the multipath delay $T_{\alpha}$ as - times the inverse of the signal bandwidth, i.e., $T_{\alpha}=2 \pi r / \Delta \omega$, where $r$ is any real number. We choose the element spacing as one half of the wavelength corresponding to the center frequency $\omega_{0}$, and the delay between taps as specified by the sampling theorem for bandpass signals. That is, $T_{o}=1 / f_{s}$ with $f_{s}=2 f_{\max } /\left\lfloor f_{\max } / \Delta f\right\rfloor$ where $\lfloor x\rfloor$ denotes the largest integer not exceeding $\boldsymbol{x}[\mathbf{1 4}]$. The mn-th element of $k j$-th submatrix of $\Phi_{d d}$ is then expressed as

$$
\begin{aligned}
{\left[\Phi_{d d_{m n}}\right]_{k j}=} & \sigma_{d}^{2} \operatorname{sinc}\left(\frac{B_{d}}{2}\left(\pi(n-m) \sin \theta_{d}+\omega_{o} T_{o}(j-k)\right)\right) \\
& \exp \left(i\left(\pi(n-m) \sin \theta_{d}+\omega_{o} T_{o}(j-k)\right)\right)
\end{aligned}
$$

The corresponding elements of $\boldsymbol{\Phi}_{\boldsymbol{s} s}$ and $\boldsymbol{\Phi}_{\boldsymbol{v}}$ can be similarly expressed.

\subsection{Dimensionality of the Source Representation Space}

As defined in [ 1], a source representation space is the span of eigenvectors associated with ' $D$ ' significant eigenvalues of the corresponding source sample covariance matrix. For example, the span of ' $D$ ' eigenvectors of $\boldsymbol{\Phi}_{d d}$ is the source representation space associated with the signal $d(t)$. The value of $D$ can also be viewed as the effective dimension of the signal subspace. If $\lambda_{j}, j=1,2, \cdots, M K$, denote the ordered eigenvalues of the matrix $\boldsymbol{\Phi}_{d d}$, then D is the smallest value for which

$$
\frac{\sum_{j=1}^{D} \lambda_{j}}{\operatorname{Trace}\left(\Phi_{d d}\right)} \geq 99.99 \%
$$

If $D_{1}$ and $D_{2}$ represent the effective dimensions of the individual signal subspaces associated with $d(t)$ and $s(t)$, then $D_{1}+D_{2}$ is the dimension of the composite signal subspace, i.e., $\left(D_{1}+D_{2}\right)$ eigenvectors of $\left(\Phi_{d d}+\Phi_{s s}\right)$ associated with $\left(D_{1}+D_{2}\right)$ ordered eigenvalues span the composite signal subspace.

\section{2 $M V$ Beamforming}

In a linearly constrained minimum variance $(M V)$ beam former, the array signals are combined so as to pass the desired directional signal to the output without distortion while maximally rejecting the signals from other directions. We consider Frost beamforme [13] with desired signal in the broad-side direction. Let $\mathbf{W}$ denote the weight vector formed by stacking the tap weights column-wise, i.e.,

$$
\mathbf{W}=\left[w_{11}, w_{21}, \cdots, w_{1 K}, w_{2 K}, \cdots, w_{M K}\right]^{T}
$$

The optimum weight vector is given by [ $\mathbf{1 3}$ ]

$$
\mathbf{W}_{\text {opt }}=\Phi^{-1} C\left[C^{T} \Phi^{-1} C\right]^{-1} \xi
$$


where $C$ is the $M K$ by $K$ constraint matrix and ? is $K$ by $\mathbf{1}$ constraint response vector as defined in [ $\mathbf{1 3}]$. The interference rejection performance of the beamformer is then specified by depth of the null in the interference direction in the output beampattern given by $\mathbf{W}_{\text {opt }}^{+} \mathbf{a}(\boldsymbol{\theta}, \boldsymbol{\omega})$, where $\mathbf{a}(\boldsymbol{\theta}, \boldsymbol{\omega})$ is the MK-dimensional steering vector at a frequency $w$.

\section{$2.3 \quad D O A$ Estimation}

As suggested in [1], we perform eigen decomposition of the prewhitened data covariance matrix, $\Phi_{\boldsymbol{v}}^{-\frac{1}{2}} \Phi\left(\Phi_{\boldsymbol{v}}^{-\frac{1}{2}}\right)^{+}$, and construct noise subspace $E_{n}$ from the eigenvectors $\mathbf{e}_{D+1}, \cdots, \mathbf{e}_{K M}$, associated with the last $K M-D$ eigenvalues of the prewhitened matrix, where $D$ is known a priori or estimated from the time-bandwidth products of the signals [ 1 ]. We note here that the noise statistics are assumed to be known a priori.

In view of the prewhitening of the data covariance matrix, the source observations are altered by the corresponding transformation. We therefore use a transformed location vector

$$
\mathbf{b}(\theta, \omega)=\Phi_{v v}^{-\frac{1}{2}} \mathbf{a}(\theta, \omega)
$$

and compute the spatial spectrum as $[6]$

$$
P(\theta)=\sum_{k=1}^{N_{f}} \frac{1}{\sum_{i=D+1}^{M K}\left|\mathbf{b}^{+}\left(\theta, \omega_{k}\right) \mathbf{e}_{i}\right|^{2}}
$$

where $N_{f}$ denotes the number of equispaced frequencies in the bandwidth of the signal over which the spectrum is averaged. Location of the peaks in $P(\theta)$ are taken as the estimates of the DOAs of the impinging sources.

\section{EFFECT OF MULTIPATH DELAY AND F-NORM OF $\left(\Phi_{d s}+\Phi_{s d}\right)$}

When the impinging signals are uncorrelated, the effective dimension of the composite signal subspace is the sum of the dimensions of the individual source representation spaces. But, when the signals are correlated as in multipath, the effective dimension falls and we refer to this as the collapse in the dimensionality of the signal subspace. The extent by which it collapses depends on the multipath delay.

We note from (2.16) and (2.17) that the correlation between the direct signal and its multipath decreases as the path delay increases. We also note that as $T_{\alpha}$ increases, the magnitude of each element of the cross-correlation matrix decreases, suggesting that we use the Fnorm of this matrix as a measure of correlation between the direct and multipath signals. In the limit, as the path delay, $T_{\alpha}$, tends to infinity, the F- norm, $\left\|\boldsymbol{\Phi}_{d s}+\Phi_{s d}\right\|_{F}$, tends to zero which corresponds to the uncorrelated case.

To illustrate the above phenomena, we considered a scenario with one signal arriving from $0^{\circ}$ and the other from $10^{\circ}, M=6$ and $K=4$. The direct signal $d(t)$ and the multipath signal $s(t)$ were assumed to arrive from $0^{\circ}$ and $10^{\circ}$, respectively. The relative bandwidth of the signals was chosen as 0.4 , and this fixed the value of To as $\omega_{a} T_{0}=2.5 \pi$ The delay of the multipath signal, $T_{\alpha}$, was taken as $\frac{2 \pi r}{\Delta \omega}$, where $\mathbf{r}$ can assume any real value. The signal and noise powers were set at $0 d B$, i.e., $\sigma_{d}^{2}=\sigma_{s}^{2}=\sigma_{v}^{2}=1$. Table 1 gives the effective dimension of the composite signal subspace, which is computed as described in the previous section. and the $\mathbf{F}$ - norm for several values of $\mathbf{r}$.

Table 1. Effect of multipath delay on $D$ and F-norm $\left(T_{\alpha}=\frac{2 \pi r}{\Delta \omega}\right)$

\begin{tabular}{|c|c|c|}
\hline$r$ & $\begin{array}{c}D \text { of }\left(\Phi_{d d}+\Phi_{s s}\right. \\
\left.+\Phi_{d s}+\Phi_{s d}\right)\end{array}$ & $\left\|\Phi_{d s}+\Phi_{s d}\right\|_{F}$ \\
\hline 0.1 & 5 & 24.21 \\
& & 19.48 \\
0.4 & 5 & 1580 \\
1 & 6 & 19.48 \\
3 & 8 & 15.89 \\
$\star$ & 8 & 3.152 \\
& & 0 \\
\hline
\end{tabular}

$$
\bar{\Phi}^{L}=\frac{1}{L} \sum_{l=1}^{L} \Phi^{l}
$$

In view of $(4.2)$, we get

$$
\bar{\Phi}^{L}=\Phi_{d d}+\Phi_{s s}+\Phi_{v v}+\frac{1}{L} \sum_{l=1}^{L}\left(\Phi_{d s}^{l}+\Phi_{s d}^{l}\right)
$$

Note that since the lag value $\tau$ increases with I ( see (4.3)) and the magnitude of the autocorrelation value decreases with $\tau$ (see (2.17)),
We note from the results of the table that though the signal subspace dimension for $\mathbf{r}=\mathbf{3}$ is same as that for the uncorrelated case, relation left between the two signals. We know from the analysis of the $M V$ beamformer for narrowband signals [11] that the interference rejection performance degrades if the interference is correlated extent of degradation depends on the ar beamformer as in the narrowband case, we used nal and $s(t)$ as the inteference. The noise power was fixed same as before, but the signal powers were set at $10 d B$ each. The optimum vector as $\xi=[1,0, \cdots, 0]^{T}$, and the beampattern was evaluated at the center frequency of the band. Note from the inset in Fig. 2 that $\mathbf{r}=\mathbf{3}$ it is still $\mathbf{1 3} \mathrm{dB}$ less than that of the uncorrelated case. This confirms our assertion that the F-norm can be used as the measure of

\section{SMOOTHING ON DECORRELATION OF THE SOURCES} (progressive smoothing) and then derive an upper and the Fis bound, we study the effect of spacprogressive smoothing. We give some numerical results to show that the bound and actual value of $\mathrm{F}$-norm have similar shapes. with $K$ taps, and with a K-tap delay line attached to each sensor. Let $\mathbf{X}^{\mathbf{l}}(t)$ denote the $K M$-dimensional stacked data vector of I- th subarray, formed from the notation of Section 2 and assuming $s(t)=d\left(t-T_{c}\right)$, the mk-th ent of $\mathbf{X}^{\prime}(t)$ can be expressed as

$$
+d\left(t-T_{\alpha}-(\mathrm{I}+\mathrm{m}-2) T_{s}-(k-1) T_{o}\right)
$$$$
+v_{l+m-1}\left(t-(k-1) T_{o}\right)
$$

The $M K$ by $M K$ sample covariance matrix of I-th subarray is then

we have shown the dependence on the position of the subarray $\mathrm{mn}$-th element in kj-th submatrix of $\boldsymbol{\Phi}_{d s}^{l}$ can be shown to be which we refer to as $L$ smoothing steps) as 


\begin{tabular}{|c|c|c|}
\hline No: of subarrays $L$ & two-source case & three-source case \\
\hline $\mathbf{1}$ & $\mathbf{5}$ & $\mathbf{5}$ \\
2 & $\mathbf{8}$ & $\mathbf{1 0}$ \\
$\mathbf{3}$ & & 12 \\
$\star$ & $\mathbf{8}$ & $\mathbf{1 2}$ \\
\hline
\end{tabular}

Note from the results of the table that the dimensionality of the signal subspace collapses when the multipath delay is small causing the impinging sources to be highly correlated, and with subarray averaging, we recover this dimensionality. In the examples considered here, the number of subarrays required for restoring the dimensionality is 2 and 3, respectievely, for the two- and three-source cases.

From (4.3) and the assumption that $d(t)$ is a bandlimited random process, we can show that

$$
\begin{gathered}
{\left[\left(\frac{1}{L} \sum_{l=1}^{L}\left(\Phi_{d s}^{l}+\Phi_{s d}^{l}\right)\right)_{m n}\right]_{k j}=} \\
\frac{\sigma_{d}^{2}}{\Delta \omega L} \int_{\omega_{o}-\Delta \omega / 2}^{\omega_{o}+\Delta \omega / 2} \exp \left(i \omega(j-k) T_{o}\right)\left(\frac{\sin \omega L\left(T_{s}-T_{d}\right) / 2}{\sin \omega\left(T_{s}-T_{d}\right) / 2}\right) \\
\left(\exp \left(i \omega\left(T_{\alpha}+(n-2) T_{s}-(m-2) T_{d}+\frac{L+1}{2}\left(T_{s}-T_{d}\right)\right)\right)\right. \\
\left.+\exp \left(-i \omega\left(T_{\alpha}+(m-2) T_{s}-(n-2) T_{d}+\frac{L+1}{2}\left(T_{s}-T_{d}\right)\right)\right)\right) d \omega
\end{gathered}
$$

where we assumed a pure delay environment for simplicity. Applying Schwarte inequality to the integrand in (4.6) and simplifying, we obtain (after some work ) (see Appendix A)

LHS of (4.6) $\leq$
Fig. 3 gives the plots of the bound (RHS of (4.8)) and the true value of the F-norm ( LHS of (4.8)), for 3 different source configurations, $\left(0^{\circ}, 10^{\circ}\right),\left(0^{\circ}, 30^{\circ}\right)$ and $\left(50^{\circ}, 60^{\circ}\right)$, as a function of the smoothing index. The plots bring out the following:

1. The bound depicts all the essential features, like the rate of fall with $L$, sidelobe nature caused by the $\frac{\sin x}{z}$ shape of the correlation function etc.

2. The rate of fall is higher when the sources are widely spaced (plots 1 and 2 )

3. The rate of fall is slower when the sources are away from the broad-side direction (plots $\mathbf{1}$ and $\mathbf{3}$ ).

As the F-norm reflects the effective correlation between the direct and multipath signals, we note from the plots that the rate of decorrelation with smoothing is faster when the sources are widely spaced and is slower when the sources are away from the broad-side direction.

In the next section, we give several simulation results which illustrate the impact of progressive smoothing on both $D O A$ estimation and $M V$ beamforming. In particular, we show that $D O A$ estimation performance improves with progressive smoothing in the finite data case and the rate of this improvement is higher in the case where the rate of fall in the F-norm is larger. We give asymptotic beampatterns for several values of $L$ to show that the interference rejection performance goes up when the norm goes down and vice versa, thus confirming our assertion that the F-norm is a measure of the effective correlation between the two signals.

\section{SIMULATION RESULTS}

To support our assertions on the impact of progressive smoothing, we conducted simulations on $D O A$ estimation and $M V$ beamforming. Fig. 4 shows the $D O A$ estimation performance for different degrees of smoothing for two senarios, $\left(0^{\circ}, \mathbf{1 0}^{\prime}\right)$ and $\left(0^{\circ}, \mathbf{3 0}\right)$. In each case, the signal and noise powers were all set at $0 d B$ and the covariance matrices were estimated from 200 snapshots of data. The value of $r$ in $\tau_{\alpha}$ was chosen as $\mathbf{0 . 4}$, and the relative bandwidth $\boldsymbol{B}_{\boldsymbol{d}}$ was fixed at $\mathbf{0 . 4}$. The spatial spectrum was computed from (2.24) choosing $N_{f}=3$.

The plots of Fig. 4 (a) show that the DOA estimation performance improves progressievely with smoothing. The plots for $L=2$, corresponding to the two scenarios, show that for the same degree of Smoothing, the rate of improvement is higher in the case of widely spaced sources. This is what we predicted from the F-norm curves of Fig. 3. Note that $D O A$ estimation fails with $L=\mathbf{1}$ in both cases.

To illustrate the effect of progressive smoothing on the interference rejection performance of the $M V$ beamformer and also to demonstrate that the residual F-norm of the cross-correlation matrix is the measure

$\left[\frac{\sigma_{d}^{2}}{L}\left(1+\frac{2 \cos \left(2 \omega_{0} T_{\alpha}+(\mathrm{m}+n-3+L) \beta\right) \sin \left(\left(B_{d} / 2\right)\left(2 \omega_{o} T_{\alpha}+(\mathrm{m}+n-3+L) \beta\right)\right.}{B_{d}\left(2 \omega_{0} T_{\alpha}+(m+n-3+L) \beta\right)}\right.\right.$

of the residual correlation between the impinging signals, we com$2 \cos (\beta L) \sin \left(\left(B_{d} / 2\right) \beta L\right)$ puted beampatterns for various degrees of smoothing and plotted the results in Fig. 5. The beampattern was evaluated at the center frequency of the band as described in Section 2.2 replacing $\Phi$ in (2.22) with $\boldsymbol{\Phi}^{L}$. The scenarios, the signal bandwidths, the multipath delay and the

$\cos \left(2 \omega_{o} T_{\alpha}+(m+n-3) \beta\right) \sin \left(\left(B_{d} / 2\right)\left(2 \omega_{o} T_{\alpha}+(m+n-3) \beta\right)\right)$

$$
B_{d}\left(2 \omega_{o} T_{\alpha}+(m+n-3) \beta\right)
$$

$-\frac{\cos \left(2 \omega_{0} T_{\alpha}+(m+n-3+2 L) \beta\right) \sin \left(\left(B_{d} / 2\right)\left(2 \omega_{o} T_{\alpha}+(m+n-3+2 L) \beta\right)\right)}{B^{\frac{1}{2}}}$

$$
\left.\left(\frac{2}{B_{d} \beta}\left(\cot \frac{\beta}{2}\left(1-\frac{B_{d}}{2}\right)-\cot \frac{\beta}{2}\left(1+\frac{R}{2}\right)\right)\right)^{\frac{1}{2}}\right]
$$

where $\beta=\omega_{o}\left(T_{s}-T_{d}\right)$. The bound on the F-norm of the smoothed cross-correlation matrix is then given by

$$
\left\|\frac{1}{L} \sum_{m=1}^{L}\left(\Phi_{d s}^{l}+\Phi_{o d}^{l}\right)\right\|_{F} \leq K \sqrt{\sum_{m=1}^{M} \sum_{n=1}^{M}(R H S o f(4.7))^{2}}
$$

subarray size were same as those used in DOA estimation (see Fig. 4) excepting that the signal powers were set at $10 d B$ each. In each case, the signal arriving from $0^{\circ}$ was assumed to be the desired signal and the multipath signal was treated as the interference. The plots of Fig. 5 depict the following.

1. There is a one-to-one correspondence between the increase ( and decrease ) in the null depth and the decrease (and increase) in the F-norm with $L$. For example, for the scenario $\left(0^{\circ}, 10^{\circ}\right)$ 
( Fig. 5(a)), the null depth progressievely increases when $L$ is increased from 1 to 4 and from 4 to 12 , but reduces for $L=15$ ( see the inset). The F-norm for the corresponding scenario (plots 1 in Fig. 3 ) shows opposite behaviour implying that the residual F-norm is the measure of the residual correlation, since the degradation in the interference rejection performance is proportional to the correlation [ 11].

2. The rate of increase in the null depth with $L$ is higher for widely spaced sources for which case the rate of fall in the F-norm is larger. This can be seen from the beampatterns for $L=1$ and 4 for the two scenarios ( see Fig. 5 ) and the corresponding F-norm plots in Fig. 3 (plots 1 and 2 ).

\section{CONCLUSIONS}

In this paper, we have shown that the effective dimension of the signal subspace collapses in the presence of multipath and it can be recovered with subarray averaging. We have proposed the Fnorm of the cross-correlation matrix as a measure of the correlation between the impinging signals and derived an upper bound on this norm which depicts all the essential features of the true F-norm. Using this bound we studied how the correlation between the direct and multipath signals falls with progressive smoothing and how the rate at which it falls depends on the spacing and directions of the sources. Computer simulations have been provided to support our theoretical predictions.

To keep the notation and the analysis simple, our analysis was restricted to two-source models. However, the results extend to the signal models with more than two sources.

\section{A APPENDIX}

Applying Schwartz inequality

$$
\int_{a}^{b} f(x) g(x) d x \leq\left(\int_{a}^{b}|f(x)|^{2} d x\right)^{\frac{1}{2}}\left(\int_{a}^{b}|g(x)|^{2} d x\right)^{\frac{1}{2}}
$$

to the integrand in Equation (4.6), we get LHS of $(4.6) \leq$

$$
\begin{gathered}
\left(\int_{\omega_{o}-\frac{\Delta \omega}{2}}^{\omega_{o}+\frac{\Delta \omega}{2}} \mid\left(\frac{\sigma_{d}^{2}}{\Delta \omega L}\right) \sin \omega L\left(\frac{T_{s}-T_{d}}{2}\right)\right. \\
\left(\exp \left(i \omega\left(T_{\alpha}+(n-2) T_{s}-(m-2) T_{d}+\frac{L+1}{2}\left(T_{s}-T_{d}\right)\right)\right)+\right. \\
\left.\left.\exp \left(-i \omega\left(T_{\alpha}+(m-2) T_{s}-(n-2) T_{d}+\frac{L+1}{2}\left(T_{s}-T_{d}\right)\right)\right)\right|^{2} d \omega\right)^{\frac{1}{2}} \\
\left(\int_{\omega_{o}-\Delta \omega / 2}^{\omega_{o}+\Delta \omega / 2}\left|\frac{1}{\sin \omega\left(\left(T_{s}-T_{d}\right) / 2\right)}\right|^{2} d \omega\right)^{\frac{1}{2}}
\end{gathered}
$$

Taking the magnitude square of the term in the first integrand of RHS of (A.1) gives

RHS of $($ A.1) $=$

$$
\begin{gathered}
\left(\int_{\omega_{0}-\Delta \omega / 2}^{\omega_{0}+\Delta \omega / 2}\left(\frac{\sigma_{d}^{2}}{\Delta \omega L}\right)^{2} \sin ^{2} \omega L\left(\frac{T_{s}-T_{d}}{2}\right)\right. \\
\left(\left(\cos \omega\left(T_{\alpha}+(n-2) T_{s}-(m-2) T_{d}+\frac{L+1}{2}\left(T_{s}-T_{d}\right)\right)\right.\right. \\
\left.+\cos \omega\left(T_{\alpha}+(m-2) T_{s}-(n-2) T_{d}+\frac{+1}{2}\left(T_{s}-T_{d}\right)\right)\right)^{2} \\
+\left(\sin \omega\left(T_{\alpha}+(n-2) T_{s}-(\mathrm{m}-2) T_{d}+\frac{L+1}{2}\left(T_{s}-T_{d}\right)\right)^{2}\right.
\end{gathered}
$$

$$
\begin{gathered}
\left.\left.-\sin \omega\left(T_{\alpha}+(m-2) T_{s}-(n-2) T_{d}+\frac{L+1}{2}\left(T_{s}-T_{d}\right)\right)\right)^{2} d \omega\right)^{\frac{1}{2}} \\
\left(\int_{\omega_{o}-\frac{A_{\omega}}{2}}^{\omega_{0}+\frac{A_{\omega}}{2}} \operatorname{cosec}^{2} \omega\left(\frac{T_{s}-T_{d}}{2}\right) d \omega\right)^{\frac{1}{2}}
\end{gathered}
$$

which can be simplified to

RHS of (A.2) =

$$
\begin{gathered}
\left(\int_{\omega_{0}-\frac{\Delta \omega}{2}}^{\omega_{0}+\frac{\Delta_{\omega}}{2}}\left(\frac{\sigma_{d}^{2}}{\Delta \omega L}\right)^{2} \sin ^{2} \omega L\left(\frac{T_{s}-T_{d}}{2}\right)\right. \\
\left.\cos ^{2} \frac{\omega}{2}\left(2 T_{\alpha}+(m+n-3+L)\left(T_{s}-T_{d}\right)\right) d \omega\right)^{\frac{1}{2}} \\
\left(\int_{\omega_{0}-\Delta \omega / 2}^{\omega_{o}+\Delta \omega / 2} \operatorname{cosec}^{2} \omega\left(\frac{T_{s}-T_{d}}{2}\right) d \omega\right)^{\frac{1}{2}}
\end{gathered}
$$

Evaluating the integral in (A.3) and simplifying , we obtain RHS of $($ A.3 $)=$

$$
\begin{gathered}
\frac{\sigma_{d}^{2}}{\Delta \omega L}\left[\left(\Delta \omega+2 \cos \left(\omega_{o}\left(2 T_{\alpha}+(m+n-3+L)\left(T_{s}-T_{d}\right)\right)\right)\right.\right. \\
\frac{\sin \left(\frac{\Delta \omega}{2}\left(2 T_{\alpha}+(m+n-3+L)\left(T_{s}-T_{d}\right)\right)\right)}{2 T_{\alpha}+(m+n-3+L)\left(T_{s}-T_{d}\right)} \\
-\frac{2 \cos \left(\omega_{o} L\left(T_{s}-T_{d}\right)\right) \sin \left(\frac{\Delta \omega}{2}\left(T_{s}-T_{d}\right)\right)}{L\left(T_{s}-T_{d}\right)} \\
-\cos \left(\omega_{0}\left(2 T_{\alpha}+(m+n-3)\left(T_{s}-T_{d}\right)\right)\right) \\
\frac{\sin \left(\frac{\Delta \omega}{2}\left(2 T_{\alpha}+(m+n-3)\left(T_{s}-T_{d}\right)\right)\right)}{2 T_{\alpha}+(m+n-3)\left(T_{s}-T_{d}\right)} \\
\frac{\cos \left(\omega_{o}\left(2 T_{\alpha}+(m+n-3+2 L)\left(T_{s}-T_{d}\right)\right)\right)}{\sin \left(\frac{\Delta \omega}{2}\left(2 T_{\alpha}+(m+n-3+2 L)\left(T_{s}-T_{d}\right)\right)\right)} \\
2 T_{\alpha}+(m+n-3+2 L)\left(T_{s}-T_{d}\right) \\
\left(\frac { 2 } { ( T _ { s } - T _ { d } ) } \left(\cot \left(\left(\frac{T_{s}-T_{d}}{2}\right)\left(\omega_{o}-\frac{\Delta \omega}{2}\right)\right)\right.\right. \\
\left.\left.\left.-\cot \left(\left(\frac{T_{s}-T_{d}}{2}\right)\left(\omega_{0}+\frac{\Delta \omega}{2}\right)\right)\right)\right)^{\frac{1}{2}}\right]
\end{gathered}
$$

Substituting $\frac{\Delta \omega}{\omega_{o}}=B_{d}$ and $\omega_{o}\left(T_{s}-T_{d}\right)=\beta$ in (A.4) gives the R.H.S of (4.7).

\section{REFERENCES}

1. Buckley, K.M., “ A Source Representation ..., ${ }^{\text {Ph.D. disserta- }}$ tion, Dept. of Elec. Eng., USC, July 1986.

2. Pisarenko, V.F., "The Retrieval ...," Geophys. J. Royal Astronomical Soc., pp. 347-366,1973.

3. Schmidt, R.O., “ Multiple Emitter ...” Proc. RADG Spec. Eat. Workshop (Griffis AFB, N.Y), pp. 243-258, 1979.

4. Bienvenu, G. and L. Kopp, "Adaptivity to …", Proc. of ICASSP, pp. 307-310, 1980.

5. Buckley, K.M., "Spatial/Spectral ..." IEEE Trans. on ASSP, pp. 249-266, March 1987.

6. Buckley, K.M. and L.J. Griffiths, “ Broad-Band ..., " IEEE Trans. on ASSP, pp. 953-964, July 1988.

7. Wang, H. and M. Kaveh, "Coherent Signal ...," IEEE Trans. on ASSP, pp. 823-831, August 1985

8. Shan, T.J. „M. Wax and T. Kailath, “ On Spatial ..., " IEEE Trans. on ASSP, pp. 806-811, August 1985.

9. Shan, T.J. and T. Kailath, "Adaptive beamforming ..., , IEEE Trans. ASSP, pp. 527-536, June 1985.

IEEE INTERNATIONAL RADAR CONFERENCE 
10. Paulraj, A., V.U. Reddy, T.J. Shan and T. Kailath, “ Performance Analysis ..., Proc. of MILCOM, pp. 41.5.1-41.5.5, 1986 .

11. Reddy, V.U., A. Paulraj and T. Kailath, " Performance Analysis ..., IEEE Trans. on ASSP, pp. 927-936, July 1987.

12. Cornpton JR, R.T., " The Bandwidth ... ," IEEE Trans. on AP, pp.5-14, January 1988.

13. Frost III, O.L. ," An Algorithm ... ," Proc. IEEE, pp. 926-935, August 1972.

14. Sam Shanmugarn, K., Digital and Analog Communication Systems, John Wiley \& Sons, p. 513, 1979.

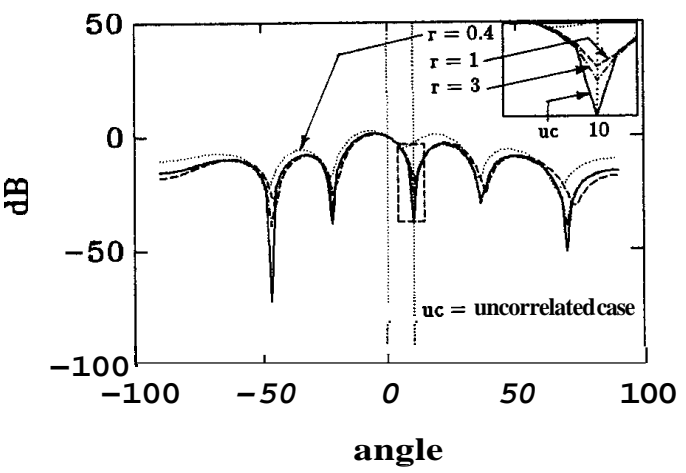

Fig. 2 Asymptotic beampatterns for different multipath delays $\left(\mathrm{M}=6, K=4, B_{d}=0.4, T_{\alpha}=2 \pi r / \Delta \omega, \mathbf{S N R}=10 d B\right.$, $\theta_{d}=0^{\circ}, \theta_{s}=10^{\circ}$ )

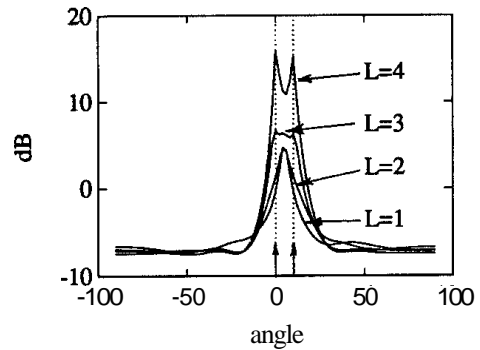

(a)

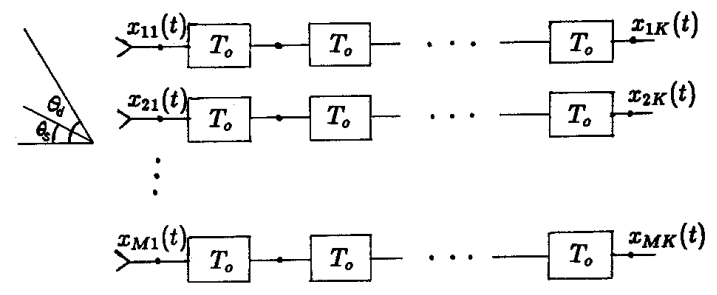

Fig. 1 A linear broadband array with $\boldsymbol{M}$ sensors and $(\boldsymbol{K}-1)$

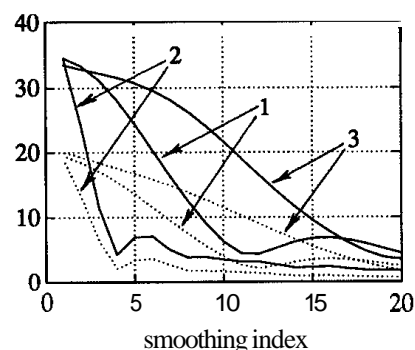

tap delays

Fig. 3 Effect of progressive smoothing on the F-norm $\left(M=6, K=4, B_{d}=0.4, r=0.4, \sigma_{d}^{2}=\sigma_{a}^{2}=\sigma_{v}^{2}=1\right)$
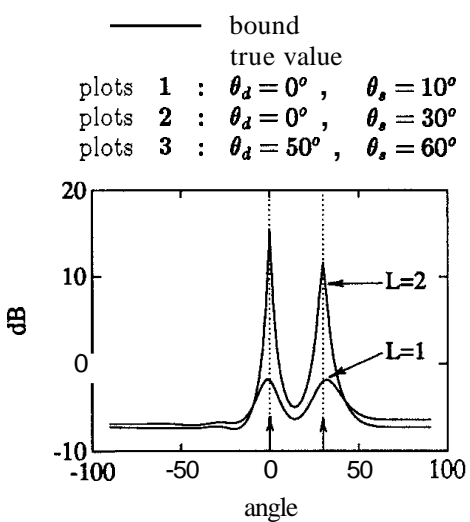

(b)

Fig. 4 Spatial spectra for different smoothing steps

$\left(\mathrm{M}=6, K=4, B_{d}=0.4, r=0.4\right.$, no. of snapshots $\left.=200\right)$

(a) $\theta_{d}=0^{\circ}, \theta_{s}=10^{\circ}$

(b) $\theta_{d}=0 ", \theta_{s}=30^{\prime \prime}$

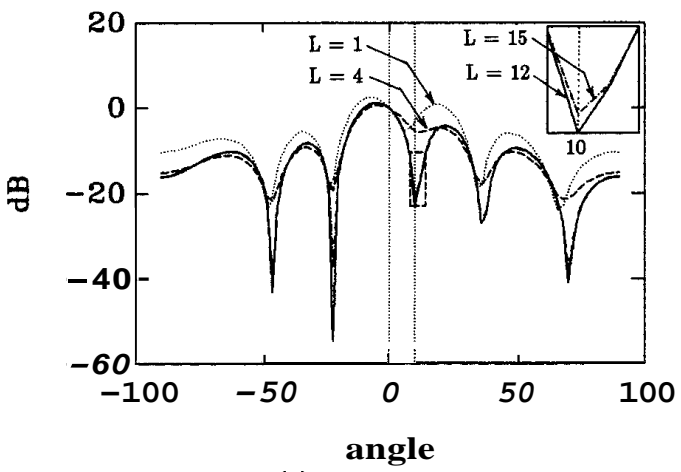

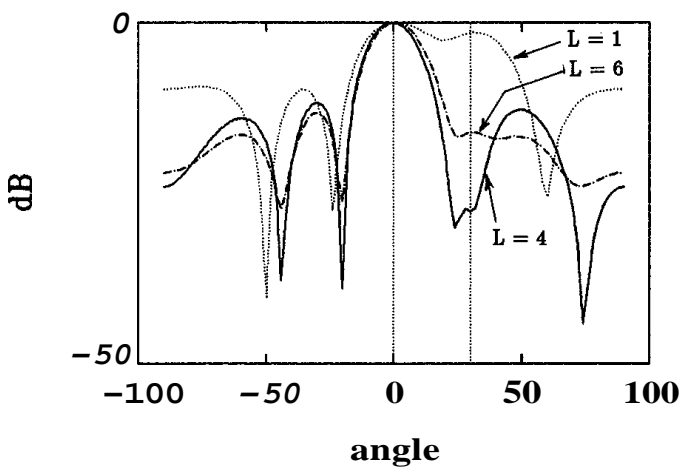

(b) $\left(M=6, K=4, B_{d}=0.4, \mathrm{r}=0.4, \sigma_{d}^{2}=\sigma_{s}^{2}=\sigma_{v}^{2}=10\right)$

(a) $\theta_{d}=0^{\circ}, \theta_{\mathrm{a}}=10^{\prime}$

537 